\title{
Decrease in field dependence following rapid eye movement sleep*
}

\author{
JOSEPH M. DE KONINCK, † DAVID KOULACK \\ and \\ GENE OCZKOWSKI \\ University of Manitoba, Winnipeg, Manitoba, Canada
}

Eight undergraduate males received the embedded figures test (EFT) on one of two testing nights in the laboratory and the rod and frame test (RFT) on the other night. Tests were presented at either the beginning or end of each of the first four rapid eye movement periods (REMPs). Test presentation nights and awakenings were counterbalanced. Performance on the RFT was better at the end of REMPs than at the onset, but a high $(\mathrm{r}=+.67)$, though not significant, correlation was found between REM density and decrement of performance on the RFT.

Since the discovery of rapid eye movement (REM) sleep (Aserinsky \& Kleitman, 1953), there has been a great deal of speculation concerning its function. Recently, attention has been focused on the possible utility of the eye movements themselves. Berger (1969) has suggested that the innervation of the oculomotor system during REM periods (REMPs) may serve to maintain facilitation of eye movements during extended periods of sleep. Subsequent work (Berger \& Scott, 1971; Berger \& Walker, 1972) has shown that oculomotor coordination and binocular depth perception are better at the end of REMPs than at the onset of REMPs and, further, that ocular imbalances are of greater magnitude and frequency at the onset of REMPs than at their termination. While oculomotor theory (Berger, 1969) refers specifically to depth perception, the present study was designed to examine the possibility that REMP activity might aid performance in other more complicated visual tasks.

To this end, the rod and frame test (RFT) and the embedded figures test (EFT) were chosen as the tasks. In the RFT the $S$ is required to adjust a rod to the vertical in a field devoid of visual cues except for a tilted frame, and in the EFT the $S$ is required to locate a simple geometric form concealed in a larger complex design. Both tests typically are used to measure the personality dimension of field dependence (Witkin et al, 1962) and,

*This research was aided by bibliographic information obtained from the UCLA Brain Information Service Sleep Bulletin and supported in part by the Medical Research Council of Canada with Grant MA-3929 to David Koulack. We thank R. J. Berger, H. J. Keselman, and J. P. Zubek for their helpful suggestions. This paper is sponsored by John P. Zubek, who takes full editorial responsibility.

†Now at the Faculty of Psychology, University of Ottawa, Ottawa, Ontario, Canada. although they have high reliability over extended periods of time (Witkin, Goodenough, \& Karp, 1967), performance on them has been altered momentarily by specific training (McAllister, 1970; Weiner, 1955), sensory isolation (Jacobson, 1966; Kurie \& Mordkoff, 1970), and arousal (Gross, 1959). In addition, there is some suggestion that the performance might be related to the nature of the eye movement sequences used to attack them (Conklin, Muir, \& Boersma, 1968; Silverman, 1968). Specifically, it was predicted that performance on the RFT and EFT would be better immediately following the end of REMPs than at their onset. In addition, it was reasoned that, if the eye movements themselves are the facilitators or indicators of innervation of the oculomotor system, there should be a direct positive correlation between measures of REMs-REM density (eye movements per unit time), total number of REMs, and duration of REMs (length of REMPs)-and performance on both the RFT and EFT.

\section{METHOD \\ Subjects}

Eight paid male undergraduate students served as Ss.

\section{Apparatus and Procedure}

Each S slept in an air-conditioned, relatively soundproof laboratory for 3 nonconsecutive nights. A standard method was used to record electroencephalograms, electrooculargrams, and electromyograms and to determine the onset and termination of REMPs (Rechtschaffen \& Kales, 1968).

The first night served as an adaptation night. On the second and third nights, Ss were awakened either at the onset or termination of each of the first four REMPs in a counterbalanced fashion by an $85-\mathrm{dB}$ buzzer.

On the second night, the EFT was administered by projecting the figures on a screen $3 \mathrm{ft}$ from the S's head and having him indicate the location of the simple figure with a 10-in. rod while sitting on the edge of the bed. Test administration procedure was standard, except that the $S$ was permitted a maximum of $60 \mathrm{sec}$ rather than $3 \mathrm{~min}$ in order to limit his time awake. Series $A$ and Series B of the EFT were divided into two sets of six figures each, and their order of presentation was counterbalanced across Ss.

On the third night, the RFT was administered using a motorized version of the portable RFT (Oltman, 1968). Four standard combinations of rod and frame tilt were presented to the $S$ in a standard manner (Witkin et al, 1962) at each of the four awakenings. The $S$ sat with his head in a headrest and made adjustments of the rod by using a bipolar switch.

On both experimental nights, the delay from awakening to the presentation of the task was approximately $90 \mathrm{sec}$ and the total time of testing was approximately $10 \mathrm{~min}$, after which the $\mathrm{S}$ was allowed to return to sleep.

\section{RESULTS AND DISCUSSION}

The mean error scores for the RFT and the mean solution times for the EFT for both onset and termination of REMPs are presented in Table 1. As 
Table 1

Mean Error on the RFT and Mean Solution Time on the EFT*

\begin{tabular}{ccccc}
\hline & \multicolumn{2}{c}{ RFT (Deg) } & \multicolumn{2}{c}{ EFT (Sec) } \\
Ss & Onset & End & Onset & End \\
\hline 1 & 4.25 & 3.18 & 65.0 & 67.7 \\
2 & 2.56 & 1.93 & 75.8 & 71.8 \\
3 & 3.50 & 2.93 & 22.0 & 28.3 \\
4 & 3.25 & 2.37 & 35.1 & 32.5 \\
5 & 2.87 & 3.12 & 30.5 & 33.2 \\
6 & $3.50 \dagger$ & 3.50 & 53.3 & 45.2 \\
7 & 1.75 & 1.34 & 12.1 & 27.6 \\
8 & 3.06 & 2.75 & 42.0 & 32.5 \\
\hline
\end{tabular}

*Lower scores indicate superior performance.

†Mean score from only one awakening. REMP onset was missed for fourth REMP.

predicted, performance on the RFT was better following REMPs than at their onset $(t=2.95, \mathrm{df}=7, \mathrm{p}<.025)$. Performance on the EFT did not differ between the two conditions. However, examination for trend disclosed improved performance on the EFT as a function of number of trials, that is, for this test a practice effect was present, overshadowing the effects of the awakening conditions $(\mathrm{F}=11.9, \mathrm{p}<.01)$.

Exploration of the relationship between RFT scores obtained after the termination of REMPs and eye movement measures revealed, contrary to expectations, a high (but not significant) correlation between REM density and decrement in RFT performance $(r=+.67)$ and low correlations between the number of eye movements and RFT scores and the duration of eye movements and RFT scores $(r=+.33, r=-.19$, respectively). To make certain that the REM density correlation was not an artifact of field-dependent Ss, a correlation was run between the mean REM density and performance on the RFT at the onset of REMP $(\mathrm{r}=+.17)$.

Although it is too early to make a definitive statement, it seems reasonable to speculate that arousal and the quality of arousal during REMPs, rather than the eye movements themselves, might play a role in performance on tasks following REMP termination. Karacan et al (1966) and Verdone (1965) have shown that REM density is related to emotionality in dreams. In our study, the generally increased arousal during REMPs might have aided post-REMP task performance, while high dream-content emotionality, as indicated by high REM density, might have had an inhibiting effect on completion of tasks presented immediately on awakening.

\section{REFERENCES}

Aserinsky, E., \& Kleitman, N. Regularly occurring periods of eye motility and concomitant phenomena during sleep. Science, 1953, 118, 273-274.

Berger, R. J. Oculomotor control: A possible function of REM sleep. Psychological Review, 1969, 76, 144-164.

Berger, R. J., \& Scott, T. D. Increased accuracy of binocular depth perception following REM sleep periods. Psychophysiology, 1971, 8, 763-768.

Berger, R. J., \& Walker, J. M. Oculomotor coordination following REM and non-REM sleep periods. Journal of Experimental Psychology, 1972, 94, 216-224.

Conklin, R. C., Muir, W., \& Boersma, F. L. Field dependency-independency and eye-movement patterns. Perceptual \& Motor Skills, 1968, 26, 59-65.

Gross, F. S. The role of set in perception of the upright. Journal of Personality, 1959, 27, 95-103.

Jacobson, G. R. Effects of brief sensory deprivation on field dependence. Journal of Abnormal Psychology, 1966, 72 , 115-118.

Karacan, J., Goodenough, D. R., Shapiro, A., \& Starker, S. Erection cycle during sleep in relation to dream anxiety. Archives of General Psychiatry, 1966, 15, 183-189.

Kurie, G. D., \& Mordkoff, A. M. Effects of brief sensory deprivation and somatic concentration of two measures of field dependence. Perceptual \& Motor Skills, 1970, 31, 683-687.

McAllister, L. W. Modification of performance on the rod-and-frame test through token reinforcement procedures. Journal of Abnormal Psychology, 1970, 75, 124-130.

Oltman, P. K. A portable rod-and-frame apparatus. Perceptual \& Motor Skills, 1968, 26, 503-506.

Rechtschaffen, A., \& Kales, A. (Eds.), A manual of standardized terminology, technique, and scoring system for sleep stages of human subjects. Washington, D.C: US Government Printing Office, 1968.

Silverman, J. Toward a more complex formulation of rod-and-frame performance in the schizophrenic. Perceptual \& Motor Skills, 1968, 27, 1111-1114.

Verdone, $P$. Temporal reference of manifest dream content. Perceptual \& Motor Skills, 1965, 20, 1253-1268.

Weiner, $M$. Effects of training in space orientation on perception of the upright. Journal of Experimental Psychology, 1955, 49, 367-373.

Witkin, H. A., Dyk, R. B., Faterson, H. F., Goodenough, D. R. \& Karp, S. A. Psychological differentiation. New Y ork: Wiley, 1962.

witkin H. Goodenough, D. R. \& Karp, S. A. Stability of cognitive style from childhood to young adulthood. Journal of Personality \& Social Psychology, 1967, 7, 291-300.

(Received for publication January 29, 1973.) 